University of Vermont

UVM ScholarWorks

$2-1-2019$

\title{
Measurement of Biodiversity (MoB): A method to separate the scale-dependent effects of species abundance distribution, density, and aggregation on diversity change
}

\author{
Daniel J. McGlinn \\ College of Charleston \\ Xiao Xiao \\ University of Maine \\ Felix May \\ Leuphana Universität Lüneburg \\ Nicholas J. Gotelli \\ University of Vermont \\ Thore Engel \\ Deutsche Forschungsgemeinschaft (DFG)
}

See next page for additional authors

Follow this and additional works at: https://scholarworks.uvm.edu/casfac

Part of the Climate Commons

\section{Recommended Citation}

McGlinn DJ, Xiao X, May F, Gotelli NJ, Engel T, Blowes SA, Knight TM, Purschke O, Chase JM, McGill BJ. Measurement of Biodiversity (MoB): A method to separate the scale-dependent effects of species abundance distribution, density, and aggregation on diversity change. Methods in Ecology and Evolution. 2019 Feb;10(2):258-69.

This Article is brought to you for free and open access by the College of Arts and Sciences at UVM ScholarWorks. It has been accepted for inclusion in College of Arts and Sciences Faculty Publications by an authorized administrator of UVM ScholarWorks. For more information, please contact scholarworks@uvm.edu. 


\section{Authors}

Daniel J. McGlinn, Xiao Xiao, Felix May, Nicholas J. Gotelli, Thore Engel, Shane A. Blowes, Tiffany M. Knight, Oliver Purschke, Jonathan M. Chase, and Brian J. McGill 


\title{
Measurement of Biodiversity (MoB): A method to separate the scale-dependent effects of species abundance distribution, density, and aggregation on diversity change
}

\author{
Daniel J. McGlinn ${ }^{1}$ (D) | Xiao Xiao ${ }^{2}$ | Felix May ${ }^{3,4}$ (D) | Nicholas J. Gotelli ${ }^{5}$ | \\ Thore Engel $^{4}$ (D) | Shane A. Blowes ${ }^{4}$ (D) | Tiffany M. Knight ${ }^{4,6,7}$ | Oliver Purschke ${ }^{4}$ (D) | \\ Jonathan M. Chase ${ }^{4,8 \dagger}$ | Brian J. McGill ${ }^{2 \dagger}$ \\ ${ }^{1}$ Biology Department, College of Charleston, Charleston, South Carolina; ${ }^{2}$ School of Biology and Ecology, and Senator George J. Mitchell Center of \\ Sustainability Solutions, University of Maine, Orono, Maine; ${ }^{3}$ Leuphana University Lüneburg, Lüneburg, Germany; ${ }^{4}$ German Centre for Integrative Biodiversity \\ Research (iDiv), Halle-Jena-Leipzig, Leipzig, Germany; ${ }^{5}$ Department of Biology, University of Vermont, Burlington, Vermont; ${ }^{6}$ Institute of Biology, Martin Luther \\ University Halle-Wittenberg, Halle (Saale), Germany; ${ }^{7}$ Department of Community Ecology, Helmholtz Centre for Environmental Research - UFZ, Halle (Saale), \\ Germany and ${ }^{8}$ Department of Computer Science, Martin Luther University, Halle-Wittenberg, Leipzig, Germany
}

Correspondence

Daniel J. McGlinn

Email: danmcglinn@gmail.com

Handling Editor: David Orme

\section{Abstract}

1. Little consensus has emerged regarding how proximate and ultimate drivers such as productivity, disturbance and temperature may affect species richness and other aspects of biodiversity. Part of the confusion is that most studies examine species richness at a single spatial scale and ignore how the underlying components of species richness can vary with spatial scale.

2. We provide an approach for the measurement of biodiversity that decomposes changes in species rarefaction curves into proximate components attributed to: (a) the species abundance distribution, (b) density of individuals and (c) the spatial arrangement of individuals. We decompose species richness by comparing spatial and nonspatial sample- and individual-based species rarefaction curves that differentially capture the influence of these components to estimate the relative importance of each in driving patterns of species richness change.

3. We tested the validity of our method on simulated data, and we demonstrate it on empirical data on plant species richness in invaded and uninvaded woodlands. We integrated these methods into a new R package (mobr).

4. The metrics that mobr provides will allow ecologists to move beyond comparisons of species richness in response to ecological drivers at a single spatial scale toward a dissection of the proximate components that determine species richness across scales.

\section{KEYWORDS}

accumulation curve, community structure, extent, grain, rarefaction curve, spatial scale, species richness, species-area curve 


\section{1 | INTRODUCTION}

Species richness - the number of species co-occurring in a specified area - is one of the most widely used biodiversity metrics. However, ecologists often struggle to understand the mechanistic drivers of richness, in part because multiple ecological processes can yield qualitatively similar effects on species richness (Chase \& Leibold, 2002; Leibold \& Chase, 2017). For example, high species richness in a local community can be maintained either by species partitioning niche space to reduce interspecific competition (Tilman, 1994), or by a balance between immigration and stochastic local extinction (Hubbell, 2001). Similarly, high species richness in the tropics has been attributed to numerous mechanisms such as higher productivity supporting more individuals, higher speciation rates and longer evolutionary time since disturbance (Rosenzweig, 1995).

Although species richness is a single metric that can be measured at a particular grain size or spatial scale, it summarizes the underlying biodiversity information that is contained in the individual organisms, each of which are assigned to a particular species, Operational Taxonomic Unit, or other taxonomic grouping. Variation in species richness can be decomposed into three components (He \& Legendre, 2002; McGill, 2010, 2011a) (a) the number and relative proportion of species in the regional source pool (i.e., the species abundance distribution, SAD), (b) the number of individuals per plot (i.e., density), and (c) the spatial distribution of individuals that belong to the same species (i.e., spatial aggregation). Changes in species richness from one place to another (or one time to another) may reflect one or a combination of all three components changing simultaneously (Chase \& Knight, 2013). Although sampling intensity and detection error influence these observations, they are also strongly influenced by experimental or observational treatments that ultimately drive the patterns of observed species richness. Thus, it is critical that we look beyond richness as a single metric, and develop methods to disentangle its underlying components that have more mechanistic links to processes (e.g., Vellend, 2016). While there are other mathematically valid decompositions of species richness and its change, the three components above (density, SAD and aggregation) are well-studied properties of ecological systems, and provide insights into mechanisms behind changes in richness and community structure (Harte, Zillio, Conlisk, \& Smith, 2008; McGlinn, Xiao, Kitzes, \& White, 2015; Supp, Xiao, Ernest, \& White, 2012). We note that the SAD and density components are also sometimes referred to as the column and row sums, respectively, of the abundance-based community matrix (e.g., Ulrich \& Gotelli, 2010).

Local richness is influenced by the shape of the regional SAD which reflects the degree to which common species dominate the individuals observed in a region, and on the total number of species in the pool. Local communities that are part of a more even regional SAD (i.e., most species having similar abundances) will have high values of local richness because it is more likely that the individuals sampled will represent different species. Local communities that are part of regions with a more uneven SAD (e.g., most individuals are a single species) will have low values of local richness because it is more likely that the individuals sampled will be the same common species ( $\mathrm{He}$ \& Legendre, 2002; McGlinn \& Palmer, 2009). The richness of the regional species pool can also influence local richness. As regional species richness increases, local richness will also increase if the local community is even a partly random subsample of the species in the regional pool. Because the regional species pool is never fully observed, the two sub-components - the shape of the SAD and the size of the regional species pool - cannot be completed disentangled. Thus, we group them together, as the SAD effect on local richness.

The number of individuals in the local community directly affects richness due to the sampling effect (the "More Individuals Hypothesis", Srivastava \& Lawton, 1998). As more individuals are sampled from the regional pool, species richness is bound to increase via sampling. This effect will be strongest at fine spatial scales; however, even at larger spatial scales, it never truly goes to zero (Palmer, McGlinn, \& Fridley, 2008; Palmer \& van der Maarel, 1995).

The spatial arrangement of individuals in a landscape is rarely random. Instead most individuals are spatially clustered or aggregated in some way, with neighbouring individuals more likely belonging to the same species. As of clusters of only a few individuals within species become more spatially clustered, local diversity will decrease because the local community or sample is likely to consist of clusters of only a few species (Chiarucci et al., 2009; Collins \& Simberloff, 2009; He \& Legendre, 2002; Karlson, Cornell, \& Hughes, 2007).

Traditionally, individual-based rarefaction has been used to control for the effect of numbers of individuals on richness comparisons (Gotelli \& Colwell, 2001; Hurlbert, 1971; Simberloff, 1972), but few methods exist (e.g., Cayuela, Gotelli, \& Colwell, 2015) for decomposing the effects of SADs and spatial aggregation on species richness. Because species richness depends intimately on the spatial and temporal scale of sampling, the relative contributions of the three components discussed above are also likely to change with scale. Spatial scale can be represented both by the numbers of samples (plots) collected, and by the number of individuals within those plots, which scales linearly with area (McGill, 2011a; see Scale and sampling effort in Methods, also see Supporting Information S7). Below, we will demonstrate that this generalized view of spatial scale allows us to synthesize the information provided by three different types of rarefaction curves: (a) spatially constrained, sample-based rarefaction, (b) nonspatial, sample-based rarefaction, and (c) (non spatial) individual-based rarefaction. Constructing these different curves allows us to parse the relative contributions of the three components of richness and how those contributions potentially change with spatial scale. Specifically, we develop a framework that provides a series of sequential analyses for estimating and testing the effects of the SAD, individual density and spatial aggregation on changes in species richness across scales. We have implemented these methods in a freely available R package mobr (https://github.com/MoBiodiv/mobr)

\section{2 | MATERIALS AND METHODS}

\section{1 | Method overview}

Our method targets data collected in standardized sampling units such as quadrats, plots, transects, net sweeps, or pit falls 
of constant area or sampling effort (we refer to these as "plots") that are assigned to treatments. We use the term treatment here generically to refer to manipulative treatments or to groups within an observational study (e.g., invaded vs. uninvaded plots). It is critical that the treatments have identical grain (i.e., area of the plots) and similar plot spatial arrangements across similar extents. If the sampling design differs between the treatments then treatment effects will be obscured by scale-mismatches; nevertheless, this can often be remedied post hoc through various types of standardization.

In an experimental study, each plot is assigned to a treatment. In an observational study, each plot is assigned to a categorical grouping variable(s). For this typical experimental/sampling design, our method provides two key outputs: (a) the observed change in richness between treatments and the relative contribution of the different components affecting richness (SAD, density, and spatial aggregation) to those changes, and (b) how species richness and its decomposition change with spatial scale. We propose two complementary ways to view scale-dependent shifts in species richness and its components: a simple-to-interpret twoscale analysis and a more informative but necessarily more complex multi-scale analysis.

The two-scale analysis provides a big-picture view of the changes between the treatments by focusing exclusively on the $\alpha$ (plot-level) and $\gamma$ (across all plots) spatial scales. It provides diagnostics for whether species richness and its components differ between treatments at the two scales. The multiscale scale analysis expands the two-scale analysis by taking advantage of three distinct types of rarefaction curves: (a) spatially constrained, sample-based rarefaction (Chiarucci et al., 2009), where the order in which plots are sampled depends on their spatial proximity (these are referred to as species accumulation curves in Gotelli \& Colwell, 2001); (b) the nonspatial, sample-based rarefaction, where individuals are randomly shuffled across plots within a treatment while maintaining average density in the plots; and (c) the individual-based rarefaction curve where again individuals are randomly shuffled across plots within a treatment, but in this case average plot density is not maintained (Gotelli \& Colwell, 2001; Hurlbert, 1971). The differences between these curves are used to isolate the effects of the SAD, density of individuals, and spatial aggregation on richness and document how these effects change as a function of scale.

\subsection{Scale and sampling effort}

Grain or the scale of interest (focus sensu Scheiner et al., 2000) can be varied by considering different numbers of individuals or plots (i.e., the sampling effort) from the $\alpha$-scale (a single plot) to the $\gamma$-scale (all plots in a treatment). It is possible to interchange numbers of individuals, numbers of plots, and spatial area because the average number of individuals accumulated scales linearly with the number of plots or area (McGill, 2011a; Supporting Information S7). This is true when considering both spatially contiguous and noncontiguous sampling designs. Theoretical treatments of the species-area relationship established the connection between the number of individuals and sampled area (Arrhenius, 1921; Harte, 2011; Hubbell, 2001; Williams, 1943). The Measurement of Biodiversity (MoB) framework uses this connection to synthesize the information provided by the three types of rarefaction curves which differ only in how they define sampling effort (i.e., scale).

\section{3 | Mathematical nomenclature}

Consider $T=2$ treatments, with $K$ replicated plots per treatment (See Supporting Information Table S1.1). Within each plot, we have measured the abundance of each species, which can be denoted by $n_{t, k, s}$, where $t=1,2$ for treatment, $k=1,2, \ldots K$ for plot number within the treatment, and $s=1,2, \ldots S$ for species identity, with a total of $S$ species recorded among all plots and treatments. The experimental design does not necessarily have to be balanced (i.e., $K$ can differ between treatments) so long as the spatial extent is similar between the treatments. However, it is important to note that inferences can only be made over a common number of individuals or samples, so large differences in sampling effort will decrease the range of scales useful for assessing treatment effects (but this can be remedied by standardization of sampling among treatments post hoc). For simplicity of notation, we describe the case of a balanced design here. The number of species observed in plot $k$ in treatment $t$ is $S_{t, k}$ (i.e., the number of species with $n_{t, k, s}>0$ ), and the number of individuals observed in plot $k$ in treatment $t$ is $N_{t, k}$ (i.e., $\sum_{s} n_{t, k, s}$ ). The spatial coordinates of each plot $k$ in treatment $t$ are $x_{t, k}$ and $y_{t, k}$. We focus on spatial patterns but our framework also applies analogously to samples distributed through time.

For clarity, we focus here on a single-factor design with two (or more) categorical treatment levels. The method can and will be extended to accommodate crossed designs and regression-style continuous treatments, which we describe in the Discussion and Supporting Information S6.

\section{4 | Two-scale analysis}

The two-scale analysis provides a simple decomposition of species richness while still emphasizing the three components and change with spatial scale. In the two-scale analysis, we compare observed species richness in each treatment and several other summary statistics at the $\alpha$-and $\gamma$-scales (Supporting Information Table S2). The summary statistics were chosen to represent the most informative aspects of individual-based rarefaction curves (Figure 1). Individual-based rarefaction curves plot the expected species richness $S_{n}$ against the number of individuals when individuals are randomly drawn from the sample at the $\alpha$ - or $\gamma$-scales. The curve can be calculated precisely using the hypergeometric sampling formula, given the SAD $\left(n_{t, k, s}\right.$ at the $\alpha$-scale, $n_{t,+, s}$ at the $\gamma$-scale) (Hurlbert, 1971).

We show how several widely used diversity metrics are represented along the individual rarefaction curve, corresponding to 


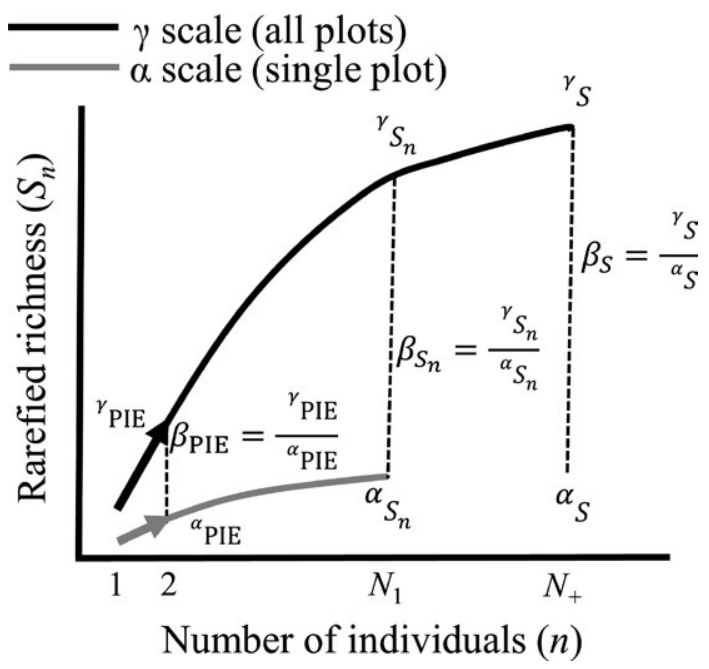

FIGURE 1 Illustration of how the key biodiversity metrics are derived from the individual-based rarefaction curves constructed at the $\alpha$ (i.e., single plot, grey line) and $\gamma$ (i.e., all plots, black line) scales. The curves are rarefied richness derived from the randomly sampling individuals from either the SAD of one or all plots. See Supporting Information Table S1.2 for definitions of metrics including ones not illustrated

$\alpha$ - and $\gamma$-scales (Figure 1, see Supporting Information S1 for detailed metric description, Chase et al. (2018) provided a more detailed description and justification of the two-scale analysis). The total number of individuals within a plot $\left(N_{t, k}\right)$ or within a treatment $\left(N_{t+}\right)$ determines the endpoint of the rarefaction curves. Rarefied richness $\left(S_{n}\right)$ controls richness comparisons for differences in individual density between treatments because it is the expected number of species for a random draw of $n$ individuals ranging from 1 to $N$. To compute $S_{n}$ at the $\alpha$-scale, we set $n$ to the minimum total number of individuals across all samples in both treatments with a hard minimum of 5 , and at the $\gamma$-scale we multiplied this $n$ value by the number of samples within a treatment (i.e., $K$ ). The SAD component is complex, but aspects of it can be understood by evaluating the relative abundances of species (e.g., via an evenness metric). Here, we use the probability of interspecific encounter (PIE) (Hurlbert, 1971) as a measure of evenness that captures differences in the relative abundances of the most common species; rare species can be examined via a number of different metrics many of which are highly correlated with S (McGill, 2011b). Importantly, PIE represents the slope at the base of the individualbased rarefaction curve (Olszewski, 2004) (Figure 1). For analyses, we convert PIE into an asymptotic effective number of species $\left(S_{\text {PIE }}\right)$ so that it can be more easily interpreted as a metric of diversity (Supporting Information S2). Whittaker's multiplicative $\beta$ diversity metrics for $S, S_{\mathrm{PIE}}$, and $S_{n}$ reflect the degree of turnover between the $\alpha$ - and $\gamma$-scales. $\beta_{S n}$ is computed at the same $n$ value at the $\alpha$ - and $\gamma$-scales (Figure 1) to control for differences in individual density and SADs. Therefore, $\beta_{S n}$ provides a means of isolating the effect of intraspecific aggregation on turnover patterns while controlling for SAD and density effects (Chase et al., 2018).
Comparison of these summary statistics between treatments identifies whether the treatments have a significant effect on richness at these two scales, and if they do, the potential proximate component(s) of the change (Chase et al., 2018). A treatment difference in $N$ implies that differences in richness may be a result of treatments changing the density of individuals. Differences in $S_{\text {PIE }}$ imply that change in the shape of the SAD may contribute to the change in richness, with $S_{\mathrm{PIE}}$ being most sensitive to changes in abundant species and $S$ being most sensitive to changes in the number of rare species. Differences in $\beta$-diversity metrics may be due to differences in any of the three components: SAD, $N$, or aggregation. $\beta_{S n}$ is unique in that it attempts to control for SAD and $N$ effects on species turnover to isolate the signal of intraspecific aggregation (Chase et al., 2018).

The treatment effect on these metrics can be visually examined with boxplots (see Empirical example section) at the $\alpha$-scale and with single points at the pooled $\gamma$-scale (unless there is replication at the $\gamma$-scale as well). Quantitative comparison of the metrics can be made with $t$-tests (ANOVAs for more than two treatments) or, for highly skewed data, nonparametric tests such as Mann-Whitney $U$ test (Kruskal-Wallis for more than two treatments).

We provide a nonparametric, randomization test where the null expectation of each metric is established by randomly shuffling the plots between the treatments, and recalculating the metrics for each reshuffle. The significance of the differences between treatments can then be evaluated by comparing the observed test statistic to the null expectation when the treatment IDs are randomly shuffled across the plots (Legendre \& Legendre, 2012). When more than two groups are compared, the test examines the overall group effect rather than specific group differences. At the $\alpha$-scale where there are replicate plots to summarize over, we use the ANOVA F-statistic as our test statistic (Legendre \& Legendre, 2012), and at the $\gamma$-scale in which we only have a single value for each treatment (and therefore cannot use the F-statistic), the test statistic is the absolute difference between the treatments (if more than two treatments are considered then it is the average of the absolute differences, $\bar{D})$. We use $\bar{D}$ as a measure of effect size at both scales.

Note that $N_{t, k}$ and $N_{t,+}$ give the same information, because one scales linearly with the other by a constant (i.e., $N_{t,+}$ is equal to $N_{t, k}$ multiplied by the number of plots $K$ within treatment). However, the other metrics $\left(S, S_{n}\right.$, and $\left.S_{\mathrm{PIE}}\right)$ are not directly additive across scales. Evaluation of these metrics at different scales may yield different insights for the treatments, sometimes even in opposite directions (Chase et al., 2018). However, complex scale-dependence may require comparison of entire rarefaction curves (rather than their two-scale summary statistics) to understand how differences in community structure change continuously across a range of spatial scales.

\section{5 | Multiscale analysis}

While the two-scale analysis provides a useful tool with familiar methods, it ignores that scale itself is not discrete, but rather is a 
continuum. Such a discrete scale perspective can thus only provide a minimal view of the scaling relationships of treatment differences. In this section, we develop a method to examine the components of change across a continuum of spatial scale. We define spatial scale by the amount of sampling effort (i.e., the number of individuals and/or the number of plots sampled; see Scale and sampling effort subsection)

\subsection{1 | The three curves}

The key innovation is to use three distinct types of rarefaction curves that capture different components of community structure. By a carefully sequenced analysis, it is possible to tease apart the effects of SAD shape, of changes in density of individuals $(N)$, and of spatial aggregation across a continuum of spatial scale. The three types of curves are summarized in Table 1. Figure 2 shows how they are constructed graphically.

The first curve is the spatial, sample-based rarefaction (sSBR) (spatially constrained rarefaction Chiarucci et al., 2009). It is constructed by accumulating plots sampled within a treatment based on their spatial position such that the most proximate plots are collected first. One can think of this as starting with a target plot and then expanding a circle centred on the target plot until one additional plot is added, then expanding the circle until another plot is added, etc. In practice, every plot is used as the starting target plot and the resulting curves are averaged to give a smoother curve. If two or more plots are of equal distance to the target plot, they are accumulated in random order.

The second curve is the nonspatial, sample-based rarefaction curve (nsSBR, Supporting Information S3). It is constructed by randomly sampling plots within a treatment, but in which the individuals in the plots have first been randomly shuffled among the plots within a treatment, while maintaining the plot-level average abundance $\left(\overline{N_{t, k}}\right)$ and the treatment-level SAD $\left(\vec{n}_{t,+}=\sum_{k} \vec{n}_{t, k}\right)$. Note that this rarefaction curve is distinct from the traditional "sample-based rarefaction curve" (Gotelli \& Colwell, 2001), in which plots are randomly shuffled to build the curve but individuals within a plot are preserved (and consequently any within-plot spatial aggregation is retained). The nsSBR contains much of the same information as the sSBR (plot density and SAD), but it has nullified any signal due to species spatial aggregation.

The third curve is the familiar individual-based species rarefaction (IBR) (Gotelli \& Colwell, 2001; Hurlbert, 1971). It is constructed by first pooling individuals across all plots within a treatment, and then randomly sampling individuals without replacement. The shape of the IBR reflects only the shape of the underlying SAD $\left(\vec{n}_{t,+}\right)$.

It can be computationally intensive to compute rarefaction curves, and therefore analytical formulations of these curves are desirable to speed up computation. It is unlikely an analytical formulation of the sSBR exists because it requires averaging over each possible ordering of nearest sites; however, analytical expectations are available for the

TAB LE 1 Summary of three types of species rarefaction curves. For treatment $t, \vec{n}_{t,+}$ is the vector of species abundances, $\vec{N}_{t}$ is the vector of plot abundances, and $\vec{d}_{t}$ is the vector of distances between plots

\begin{tabular}{|c|c|c|c|c|}
\hline Curve Name & Acronym & Notation & Method for accumulation & Interpretation \\
\hline $\begin{array}{l}\text { Spatial, sample-based } \\
\text { rarefaction curve }\end{array}$ & sSBR & $\mathrm{E}\left[S_{t} \mid k, \vec{n}_{t,+}, \vec{N}_{t}, \vec{d}_{t}\right]$ & $\begin{array}{l}\text { Spatially explicit sampling in } \\
\text { which the most proximate } \\
\text { plots to a focal plot are } \\
\text { accumulated first. All possible } \\
\text { focal plots are considered and } \\
\text { the resulting curves are } \\
\text { averaged over }\end{array}$ & $\begin{array}{l}\text { The sSBR includes all information in the data } \\
\text { including effect of SAD, effect of density of } \\
\text { individuals, and effect of spatial aggregation }\end{array}$ \\
\hline $\begin{array}{l}\text { Nonspatial, sample-based } \\
\text { rarefaction curve }\end{array}$ & $\mathrm{nsSBR}$ & $\mathrm{E}\left[S_{t} \mid k, \vec{n}_{t,+}, \vec{N}_{t}\right]$ & $\begin{array}{l}\text { Random sampling of } k \text { plots } \\
\text { after removing intraspecific } \\
\text { spatial aggregation by } \\
\text { randomly shuffling individuals } \\
\text { across plots while maintaining } \\
\text { average plot-level abundance } \\
\left(\overline{N_{t, k}}\right) \text { and the treatment-level } \\
\mathrm{SAD}\left(\mathrm{n}_{t,+, s}=\sum_{k} n_{t, k, s}\right) \text {. We use an } \\
\text { analytical extension of the } \\
\text { hypergeometric distribution } \\
\text { demonstrating this curve is a } \\
\text { rescaling of the IBR based on } \\
\text { the ratio: (average density } \\
\text { across treatments)/(average } \\
\text { density of treatment of } \\
\text { interest) }\end{array}$ & $\begin{array}{l}\text { The nsSBR reflects both the shape of the SAD } \\
\text { and the difference in density between the } \\
\text { treatments. If density between the two } \\
\text { treatments is identical then this curve } \\
\text { converges on the IBR }\end{array}$ \\
\hline $\begin{array}{l}\text { Individual-based } \\
\text { rarefaction curve (IBR) }\end{array}$ & IBR & $\mathrm{E}\left[S_{t} \mid N, \vec{n}_{t,+}\right]$ & $\begin{array}{l}\text { Random sampling of } N \\
\text { individuals from the observed } \\
\operatorname{SAD}\left(\vec{n}_{t,+}\right) \text { without replacement }\end{array}$ & $\begin{array}{l}\text { By randomly shuffling individuals with no } \\
\text { reference to plot density, all spatial and } \\
\text { density effects are removed. Only the effect } \\
\text { of the SAD remains }\end{array}$ \\
\hline
\end{tabular}




\section{(a) Spatial, sample-based rarefaction (sSBR)}

Accumulate plots by nearest neighbors
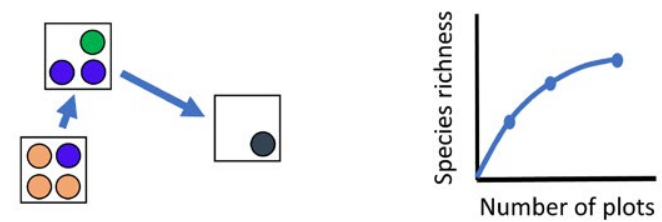

(b) Non-spatial, sample-based rarefaction (nsSBR)

Shuffle individuals between plots retaining density, then accumulate plots randomly (breaking spatial structure)

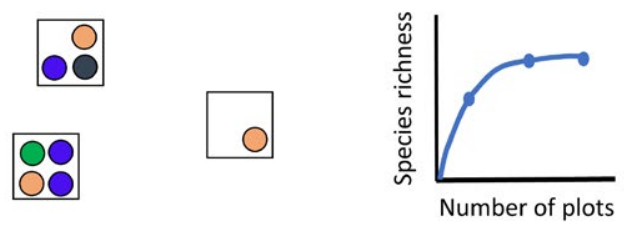

\section{(c) Individual-based rarefaction (IBR)}

Pool individuals across plots within a treatment, then accumulate individuals randomly (breaking density and spatial effects)
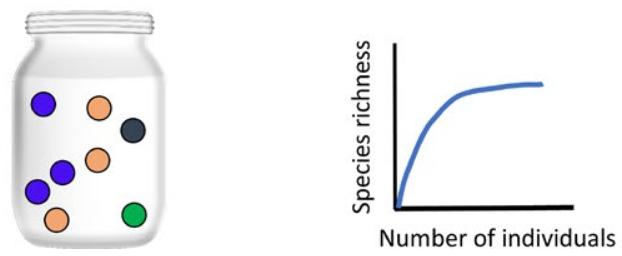

FIGURE 2 Illustration of how the three rarefaction curves are constructed. Circles of different colours represent individuals of different species. See Table 1 for detailed description of each rarefaction curve

nsSBR and IBR. Specifically, we used the hypergeometic formulation provided by Hurlbert (1971) to estimate expected richness of the IBR. To estimate the nsSBR we extended Hurlbert's (1971) formulation (see Supporting Information S3). Our derivation demonstrates that the nsSBR is a rescaling of the IBR based upon the degree of difference in density between the two treatments under consideration. Specifically, we use the ratio of average community density to the density in the treatment of interest to rescale sampling effort in the individual based rarefaction curve. For a balanced design, the individual rarefaction curve of Treatment 1 can be adjusted for density effects by multiplying the sampling effort of interest by: $\left(\sum_{t} \sum_{k} N_{t, k}\right) /\left(2 \cdot \sum_{k} N_{1, k}\right)$. Similarly, the Treatment 2 curve would be rescaled by $\left(\sum_{t} \sum_{k} N_{t, k}\right) /\left(2 \cdot \sum_{k} N_{2, k}\right)$. If the treatment of interest has the same density as the average community density then there is no density effect, and the nsSBR is equivalent to the IBR. Here, we have based the density rescaling on average number of individuals, but alternatives exist, such as using maximum or minimum treatment density. Note that the nsSBR is only relevant in a treatment comparison that contrasts with the other two rarefaction curves, which can be constructed independently of any consideration of treatment effects.

\subsection{2 | The mechanics of isolating the distinct effects of spatial aggregation, density and SAD}

The three curves capture different components of community structure that influence richness changes across scales (measured in number of samples or number of individuals, both of which can be easily converted to area, Table 1). Therefore, if we assume the components contribute additively to richness, then the effect of a treatment on richness propagated through a single component at any scale can be obtained by subtracting the rarefaction curves from each other. For simplicity and tractability, we assume additivity to capture first-order effects. This assumption is supported by Tjørve, Kunin, Polce, and Tjørve's (2008) demonstration that an additive partitioning of richness using rarefaction curves reveals random sampling and aggregation effects when using presence-absence data. We further validated this assumption using sensitivity analysis (see Supporting Information S5). Below we describe the algorithm to obtain the distinct effect of each component. Figure 5 provides a graphic illustration.

\section{Effect of aggregation}

The difference between the sSBRs of two treatments $\Delta\left(S_{21} \mid k, \vec{n}_{t,+}, \vec{N}_{t}, \vec{d}_{t}\right)=\mathrm{E}\left[S_{2} \mid k, \vec{n}_{2,+}, \vec{N}_{2}, \vec{d}_{2}\right]-\mathrm{E}\left[S_{1} \mid k, \vec{n}_{1,+}, \vec{N}_{1}, \vec{d}_{1}\right]$, gives the observed difference in richness between treatments across scales (Figure 5a,d, light-green shaded area and solid curve respectively). It encapsulates the treatment effect propagated through all three components: shape of the SAD, density of individuals, and spatial aggregation. Differences between treatments in any of these factors could potentially translate into observed difference in species richness.

Similarly, the difference between the nsSBRs $\Delta\left(S_{21} \mid k, \vec{n}_{t,+}, \vec{N}_{t}\right)=E\left[S_{2} \mid k, \vec{n}_{2,+}, \vec{N}_{2}\right]-E\left[S_{1} \mid k, \vec{n}_{1,+}, \vec{N}_{1,}\right.$, gives the expected difference in richness across treatments when spatial aggregation is removed (Figure 5b,e, light-green shaded area and dashed curve respectively). The distinct effect of aggregation across treatments from one plot to $k$ plots can thus be obtained by taking the difference between the two $\Delta S$ values (Figure $5 \mathrm{~g}$,i, light-blue shaded area and solid line respectively), i.e.,

$$
\begin{aligned}
& \Delta\left(S_{21}\right. \text { |aggregation) } \\
& =\Delta\left(S_{21} \mid k, \vec{n}_{t,+}, \vec{N}_{t}, \vec{d}_{t}\right)-\Delta\left(S_{21} \mid k, \vec{n}_{t,+}, \vec{N}_{t}\right) \\
& =\underbrace{\left.E\left[S_{2} \mid k, \vec{n}_{2,+}, \vec{N}_{2}, \vec{d}_{2}\right]-E\left[S_{1} \mid k, \vec{n}_{1,+}, \vec{N}_{1}, \vec{d}_{1}\right]\right)}_{\text {effect of aggregation, density, and SAD }}-\underbrace{\left.E\left[S_{2} \mid k, \vec{n}_{2,+}, \vec{N}_{2}\right]-E\left[S_{1} \mid k, \vec{n}_{1,+}, \vec{N}_{1}\right]\right)}_{\text {effect of density and SAD }}
\end{aligned}
$$

Equation 1 demonstrates that the effect of aggregation can be thought of as the difference between treatment effects quantified by the sSBR and the nsSBR curves. An algebraic rearrangement of Equation 1 demonstrates that can $\Delta\left(S_{21}\right.$ laggregation) also be thought of as the difference between the treatments of the same type of rarefaction curve:

$$
=\underbrace{\left(E\left[S_{2} \mid k, \vec{n}_{2,+}, \vec{N}_{2}, \vec{d}_{2}\right]-E\left[S_{2} \mid k, \vec{n}_{2,+}, \vec{N}_{2}\right]\right)}_{\text {effect of aggregation in Treatment 2 }}-\underbrace{\left(E\left[S_{1} \mid k, \vec{n}_{1,+}, \vec{N}_{1}, \vec{d}_{1}\right]-E\left[S_{1} \mid k, \vec{n}_{1,+}, \vec{N}_{1}\right]\right)}_{\text {effect of aggregation in Treatment 1 }}
$$


This simple duality can be extended to the estimation of the density and SAD effects, but we will only consider the approach laid out in Equation 1 below. In Figure 5, we separate each individual effect using the approach of Equation 1 while the code in the mobr package uses the approach of Equation 2.

One thing to note is that the effect of aggregation always converges to zero at the maximal spatial scale ( $k=K$ plots) for a balanced design. This is because when all plots have been accumulated $\Delta\left(S_{21} \mid k, \vec{n}_{t,+}, \vec{N}_{t}, \vec{d}_{t}\right)$, and $\Delta\left(S_{21} \mid k, \vec{n}_{t,+}, \vec{N}_{t}\right)$ will both converge on the difference in total richness between the treatments. However, for an unbalanced design in which one treatment has more plots than the other, $\Delta\left(S_{21}\right.$ laggregation) would converge to a nonzero constant because $\mathrm{E}\left[S_{t} \mid k, \vec{n}_{t,+}, \vec{N}_{t}, \vec{d}_{t}\right]-\mathrm{E}\left[S_{t} \mid k, \vec{n}_{t,+}, \vec{N}_{t}\right]$ would be zero for one treatment but not the other at the maximal spatial scale (i.e., $\min \left(K_{1}, K_{2}\right)$ plots). This artefact is inevitable and should not be interpreted as a real decline in the relative importance of aggregation on richness, but simply as the diminishing ability to detect aggregation without sampling a larger region.

\section{Effect of density}

In the same vein, the difference between the IBRs of the two treatments, $\Delta\left(S_{21} \mid N, \vec{n}_{t,+}\right)=E\left[S_{2} \mid N, \vec{n}_{2,+}\right]-E\left[S_{1} \mid N, \vec{n}_{1,+}\right]$, yields the treatment effect on richness propagated through the shape of the SAD alone, with the other two components removed (Figure $5 \mathrm{c}$,f, light-green shaded area and dotdashed curve respectively). The distinct effect of density across treatments from one individual to $N$ individuals can thus be obtained by subtracting the $\Delta S$ value propagated through the shape of the SAD alone from the $\Delta S$ value propagated through the compound effect of the $S A D$ and density (Figure 5h,j, light-blue shaded area and solid line respectively), i.e.,

$$
\begin{aligned}
& \Delta\left(S_{21} \mid \text { density }\right)=\Delta\left(S_{21} \mid N, \vec{n}_{t,+}, \vec{N}_{t}\right)-\Delta\left(S_{21} \mid N, \vec{n}_{t,+}\right) \\
& =\underbrace{\left(E\left[S_{2} \mid N, \vec{n}_{2,+}, \vec{N}_{2}\right]-E\left[S_{1} \mid N, \vec{n}_{1,+}, \vec{N}_{1}\right]\right)}_{\text {effect of density and } S A D}-\underbrace{\left(E\left[S_{2} \mid N, \vec{n}_{2,+}\right]-E\left[S_{1} \mid N, \vec{n}_{1,+}\right]\right)}_{\text {effect of SAD }}
\end{aligned}
$$

Note that in Equation 3, spatial scale is defined with respect to numbers of individuals sampled $(N)$ (and thus the grain size that would be needed to achieve this) rather than the number of samples ( $k$ ).

\section{Effect of SAD}

The distinct effect of the shape of the SAD on richness between the two treatments is simply the difference between the two IBRs
(Figure 5c,f,k, light-green shaded area, dot-dashed curve, and lightblue solid curve, respectively), i.e.,

$$
\Delta\left(S_{21} \mid \mathrm{SAD}\right)=\Delta\left(S_{2} \mid p, \vec{n}_{2,+}\right)-\Delta\left(S_{1} \mid p, \vec{n}_{1,+}\right)
$$

The scale of $\Delta\left(S_{21} \mid S A D\right)$ ranges from one individual, where both individual rarefaction curves have one species and thus $\Delta\left(S_{21} \mid S A D\right)=0$, to $N_{\min }=\min \left(N_{1,+}, N_{2,+}\right)$, which is the lower total abundance between the treatments.

The formulae used to identify the distinct effect of the three factors are summarized in Table 2.

\subsection{3 | Significance tests and acceptance intervals}

In the multiscale analysis, we also applied Monte Carlo permutation procedures to 1) construct acceptance intervals (or nonrejection intervals) across scales on simulated null changes in richness, and 2) carry out goodness-of-fit tests on each component (Loosmore \& Ford, 2006; Diggle-Cressie-Loosmore-Ford test [DCLF]; Baddeley et al., 2014). See Supporting Information S4 for descriptions of how each set of randomizations was developed to generate $95 \%$ acceptance intervals $\left(\Delta S_{\text {null }}\right)$, which can be compared to the observed changes $\left(\Delta S_{\text {obs }}\right)$. Strict interpretations of significance in relation to the acceptance intervals is not warranted because each point along the spatial scale ( $x$-axis) is effectively a separate comparison. Consequently, a problem arises with multiple nonindependent tests and the $95 \%$ bands cannot be used for formal significance testing due to Type I errors. The DCLF test (see Supporting Information S4) provides an overall significance test with a proper Type I error rate (Loosmore \& Ford, 2006), but this test in turn suffers from Type II error (Baddeley et al., 2014). There is no mathematical resolution to this and user judgement should be emphasized if formal $p$-values are needed.

\section{6 | Sensitivity analysis}

We tested the validity of our approach (and the significance tests) by simulations using the R package mobsim (May, Gerstner, McGlinn, Xiao, \& Chase, 2018). The goal was to establish the rate of type I error (i.e., detecting significant treatment effect through a component when it does not differ between treatments) and type II error (i.e., nonsignifi-

\begin{tabular}{|c|c|c|}
\hline Factor & Formula & Note \\
\hline Aggregation & $\Delta\left(S_{21}\right.$ |aggregation $)=\Delta\left(S_{21} \mid k, \vec{n}_{t,+}, \vec{N}_{t}, \vec{d}_{t}\right)-\Delta\left(S_{21} \mid k, \vec{n}_{t,+}, \vec{N}_{t}\right)$ & $\begin{array}{l}\text { Artificially, this effect always converges to zero at the } \\
\text { maximal spatial scale ( } K \text { plots) for a balanced design, or } \\
\text { a nonzero constant for an unbalanced design }\end{array}$ \\
\hline Density & $\Delta\left(S_{21} \mid\right.$ density $)=\Delta\left(S_{21} \mid N, \vec{n}_{t,+}, \vec{N}_{t}\right)-\Delta\left(S_{21} \mid N, \vec{n}_{t,+}\right)$ & $\begin{array}{l}\text { To compute this quantity, the } x \text {-axes of the nsSBR are } \\
\text { converted from plots to individuals using average } \\
\text { individual density }\end{array}$ \\
\hline SAD & $\Delta\left(S_{21} \mid S A D\right)=\Delta\left(S_{2} \mid N, \vec{n}_{2,+}\right)-\Delta\left(S_{1} \mid N, \vec{n}_{1,+}\right)$ & $\begin{array}{l}\text { This is estimated directly by comparing the IBRs } \\
\text { between two treatments }\end{array}$ \\
\hline
\end{tabular}
cant treatment effect through a component when it does differ). This

TAB LE 2 Calculation of effect size curves 
was achieved by systematically comparing simulated communities in which we altered one or more components while keeping the others unchanged (see Supporting Information S5). Overall, the benchmark performance of our method was good. When a factor did not differ between treatments, the detection of significant difference was low (Supporting Information Table S5.1). Conversely, when a factor did differ, the detection of significant difference was high, but decreased at smaller effect sizes. Thus, we were able to control both Type I and Type II errors at reasonable levels. In addition, there did not seem to be strong interactions among the components - the error rates remained consistently low even when two or three components were changed simultaneously. The code to carry out the sensitivity analysis run the R script $/$ scripts/mobr_sensitivity.R archived on Zenodo (McGlinn, Xiao, May, Engel, \& Oliver, 2018)

\section{3 | AN EMPIRICAL EXAMPLE}

In this section, we illustrate the potential of our method with an empirical example presented in Powell, Chase, and Knight (2013). Invasion of an exotic shrub, Lonicera maackii, has caused significant, but strongly scale-dependent, decline in the diversity of understorey plants in eastern Missouri (Powell et al., 2013). Specifically, Powell et al. (2013) showed that the effect size of the invasive plant on herbaceous plant species richness was relatively large at plot-level spatial scales $\left(1 \mathrm{~m}^{2}\right)$, but the proportional effect declines with increasing windows of observations, with the effect becoming negligible at the largest spatial scale $\left(500 \mathrm{~m}^{2}\right)$. Using a null model approach, the authors further identified that the negative effect of invasion was mainly due to the decline in plant density observed in invaded plots. To recreate these analyses run the $\mathrm{R}$ script $\sim /$ scripts/methods ms figures. $\mathrm{R}$ archived on Zenodo (McGlinn et al., 2018)

The original study examined the effect of invasion across scales using the slope and intercept of the species-area relationship. We now apply our MoB approach to data from one of their sites from Missouri, where the numbers of individuals of each species were recorded from $501-\mathrm{m}^{2}$ plots sampled from within a $500-\mathrm{m}^{2}$ region in the invaded part of the forest, and another 50 plots from within a $500-\mathrm{m}^{2}$ region in an adjacent uninvaded part of the forest. Our method leads to conclusions that are qualitatively similar to the original study, but with a richer analysis of the underlying components and their scale dependence. Moreover, our methods show that invasion influenced both the SAD and spatial aggregation, in addition to density, and that these effects went in opposite directions that depended on the spatial scale.

Invasion decreased total abundance ( $N$, Figure 3, $p=0.001)$, suggesting a possible influence on $S$. Indeed, the two-scale analysis suggests that invasion decreases average richness $(S)$ at the $\alpha$ (Figure 4a, $\bar{D}=5.2, p=0.001$ ) but not $\gamma$-scale (Figure 4c, $\bar{D}=16, p=0.447$ ). Comparing rarefied richness $\left(S_{n}\right.$, Figure $\left.4 d, f\right)$ allows us to test if the negative influence of invasion on $N$ influenced $S$ directly. Specifically, we found $S_{n}$ was higher in the invaded areas (significantly so $\bar{D}=15.59$, $p=0.001$ at the $\gamma$-scale evaluated at $n=250$; Figure 4f), which indicates
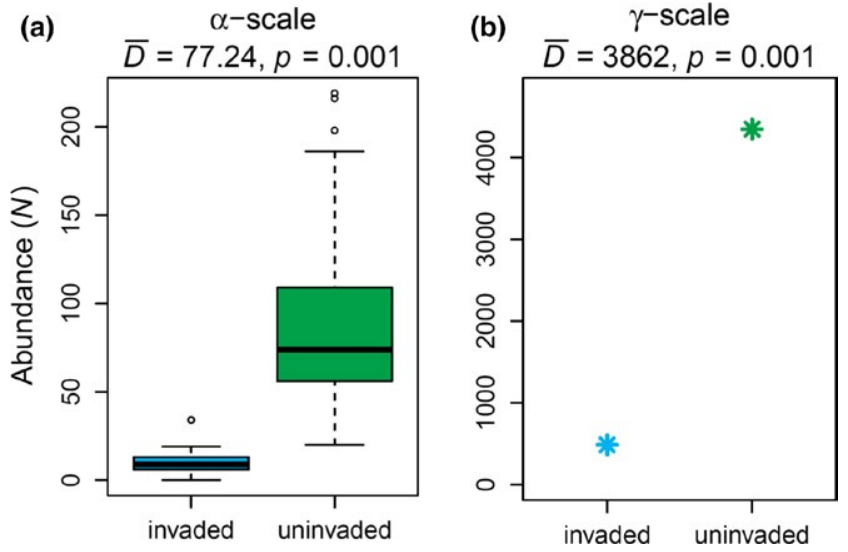

FIGURE 3 The total abundance $(N)$ of vascular plant species in plots invaded (blue boxplots and points) and uninvaded (green boxplots and points) by Lonicera maackii at the $\alpha$-scale (a, single plot) and the $\gamma$-scale (b, all plots). The $p$-values are based on 999 permutations of the treatment labels

that once the negative effect on abundance was controlled, invasion actually increased diversity through an increase in species evenness.

To identify whether the increase in evenness due to invasion was primarily because of shifts in common or rare species, we examined ENS of PIE $\left(S_{\mathrm{PIE}}\right)$ which is more sensitive to common species relative to comparisons of $S$, which is more sensitive to rare species. At the $\alpha$ - and $\gamma$-scales, invasion increased evenness in the common species (Figure 4g,h). In other words, the degree of dominance by any one species was reduced in the invaded sites.

The $\beta$ diversity metrics were significantly higher (Figure 4b,e,h, $p=0.001)$ in the invaded sites, suggesting that invaded sites had greater spatial species turnover and thus were more heterogeneous. These increases in spatial turnover appeared to be only slightly due to the sole effect of increased spatial intraspecific aggregation in invaded sites as $\beta_{S n}$ displayed the most modest effect size $(\bar{D}=0.4)$. Therefore, it appears that the shift in SAD and decreased $N$ also are playing a role in increasing $\beta$-diversity in the invaded treatment as they are reflected in $\beta_{S}$ and $\beta_{\text {SPIE }}$.

Overall the two-scale analysis indicates: (a) that there are scaledependent shifts in richness, (b) that these are caused by invasion decreasing $N$, increasing evenness in common species and increasing species patchiness.

Applying the multiscale analysis, we further disentangled the effect of invasion on diversity through the three components (SAD, density, and aggregation) across all scales of interest. Figure $5 a-c$ present the three sets of curves for the two treatments: the sSBR, in which plots accumulate by their spatial proximity (Figure 5a); the nsSBR, in which individuals are randomized across plots within a treatment (Figure 5b); and the IBR, in which species richness is plotted against number of individuals (Figure $5 c$ ). Figure $5 d-f$ show the effect of invasion on richness, obtained by subtracting the green curve from the blue curve for each pair of curves. The bottom panels ( $i-k)$, show the effect of invasion on richness through each of the three factors, is obtained by subtracting the curves in panels $g$ and $h$ from each other. The 

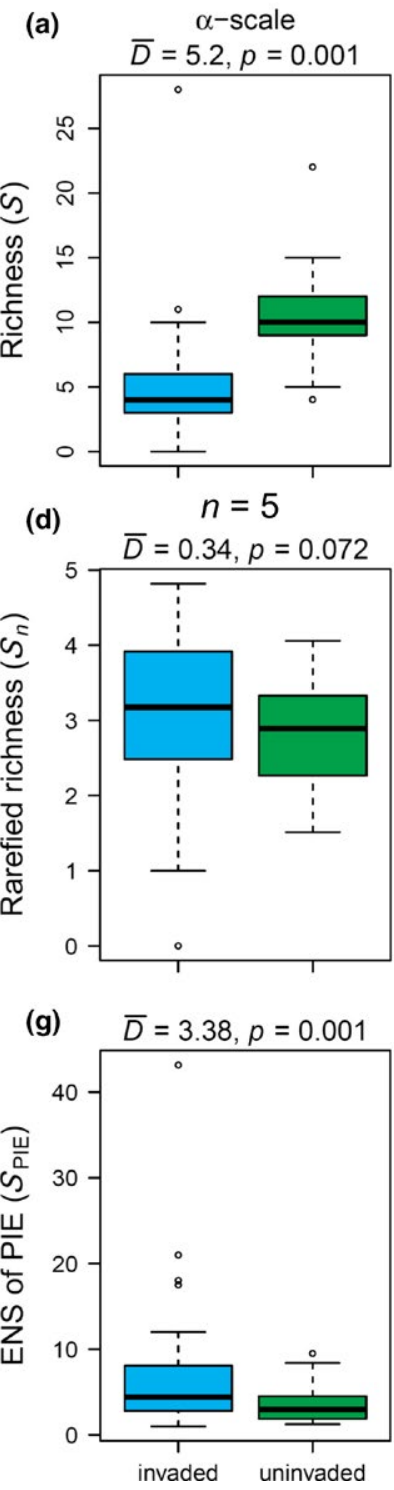
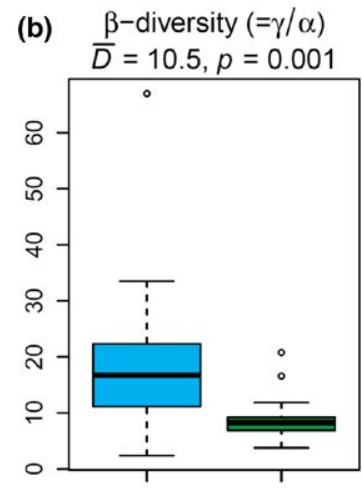

(e) $n=5$
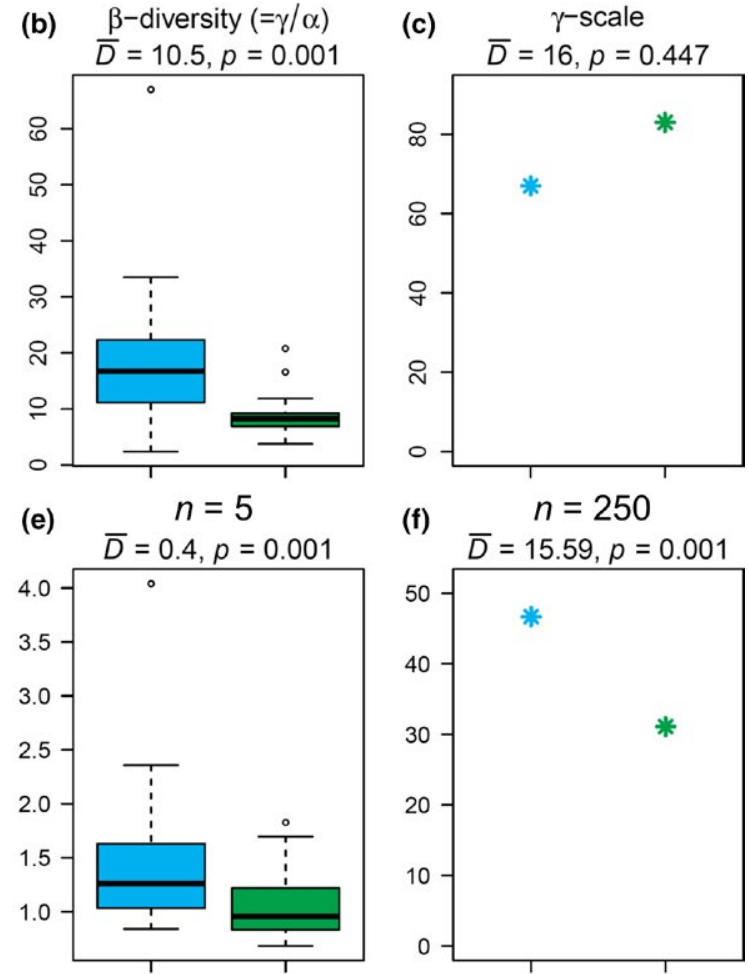

(f)

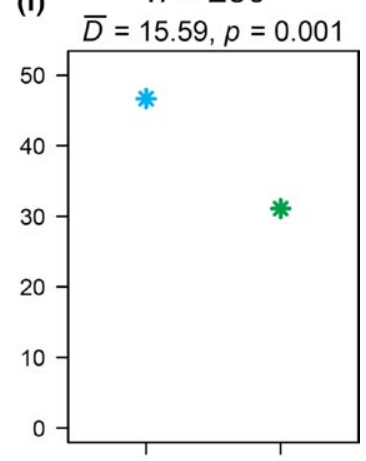

(h) $\bar{D}=1.32, p=0.001$

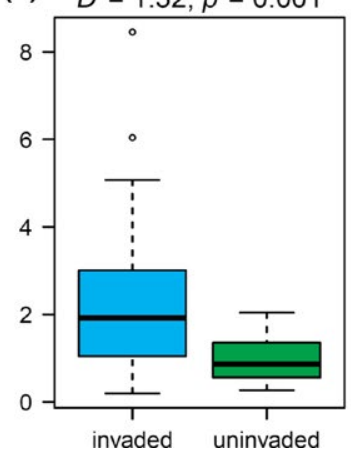

(i) $\bar{D}=5.9, p=0.001$

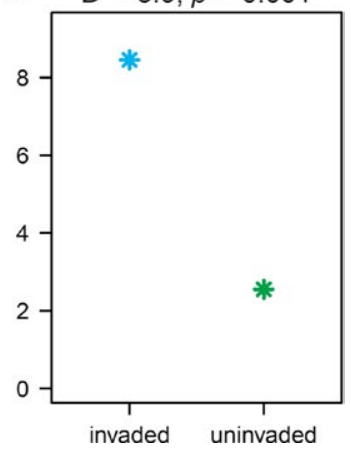

FIGURE 4 The two-scale analysis for the biodiversity metrics of the invaded (blue boxplots and points) and uninvaded (green boxplots and points) treatments at the $\alpha$ (i.e., single plot), $\beta$ (i.e., between plots), and $\gamma$ (i.e., all plots) scales. Rarefied richness $\left(S_{n}\right.$, panels $\left.d-f\right)$ were computed for $n=5,5$, and 250 individuals for the $\alpha$ (d), $\beta$ (e), and $\gamma$ (f) scales respectively. The $p$-values are based on 999 permutations of the treatment labels contribution of each component to difference in richness between the invaded and uninvaded sites is further illustrated in Figure 5.

Consistent with the original study, our approach shows that the invaded site had lower richness than the uninvaded site at all scales (Figure 5a). Separating the effect of invasion into the three components, we find that invasion actually had a positive effect on species richness through its impact on the shape of the SAD (Figures 5k and $6 a)$, which contributed to approximately $20 \%$ of the observed change in richness (Figure 6b). This counter-intuitive result suggests that invasion has made the local community more even, meaning that the dominant species were most significantly influenced by the invader. However, this positive effect was completely overshadowed by the negative effect of invasion on species richness through reductions in the density of individuals (Figures 5, and 6a), which makes a much larger contribution to the effect of invasion on richness (as large as $80 \%$, Figure $6 \mathrm{~b}$ ). Thus, the most detrimental effect of invasion was the sharp decline in the number of individuals. The effect of aggregation (Figure $5 \mathrm{i}$ ) is much smaller compared with the other two components and was most important at small spatial scales. Our approach thus validates the findings in the original study but provides a more comprehensive way to quantify the contribution to richness decline caused by invasion by each of the three components, across spatial scales.

\section{4 | DISCUSSION}

How does species richness differ between experimental conditions or among sites that differ in key parameters in an observational study? This fundamental question in ecology often lacks a simple answer, because the magnitude (and sometimes even the direction) of change in richness is strongly influenced by spatial scale (Blowes, Belmaker, \& Chase, 2017; Chalcraft, Williams, Smith, \& Willig, 2004; Fridley, Brown, \& Bruno, 2004; Knight \& Reich, 2005; Palmer et al., 2008; Powell et al., 2013; Chase et al., 2018). Species richness is proximally determined by three underlying components - SAD, density and aggregation - which are also scale-dependent; this obscures 
FIGURE 5 Application of the Measurement of Biodiversity (MoB) multiscale analysis to the invasion dataset. Panels a-c, show the invaded (blue) and uninvaded (green) in the three types of rarefaction curves (defined in Fig. 2 and Table 1). The light-green polygons represent the difference (i.e., treatment effect) for each set of curves which is plotted in panels d-f. By taking the difference again (light-blue shaded area and curves in $g$ and $h$ ) we can obtain the treatment effect on richness through a single component (i-k). See text for details (Equation 1). The grey shaded area in i-k shows the $95 \%$ acceptance interval for each null model, the cross-scale DCLF test for each factor was significant $(p=0.001)$. The dashed line shows the point of no-change in richness between the treatments. Note that in panel c) the IBR are only shown across their common range of individuals (a)

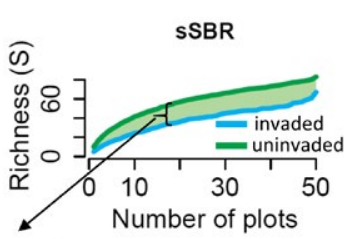

Difference in $\mathrm{S}$

(d)

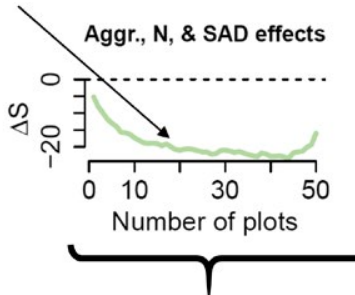

(g)

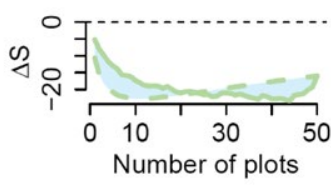

(i)

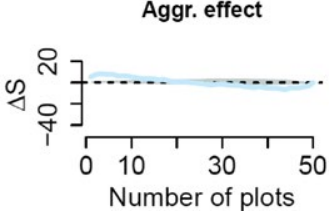

(b)

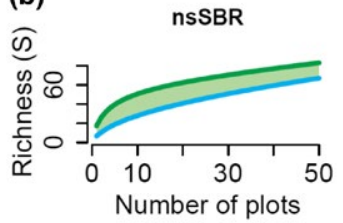

(e)
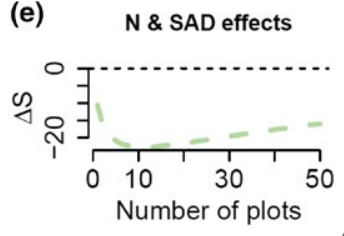

(h)

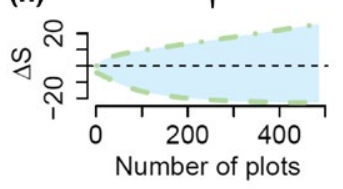

(j)

$\mathrm{N}$ effect

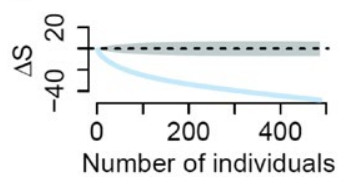

(c)

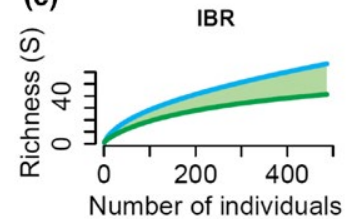

(f)

SAD effect

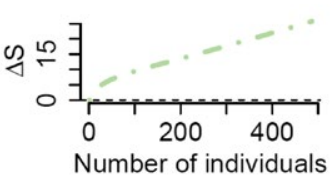

SAD effect Number of Individuals

(a)

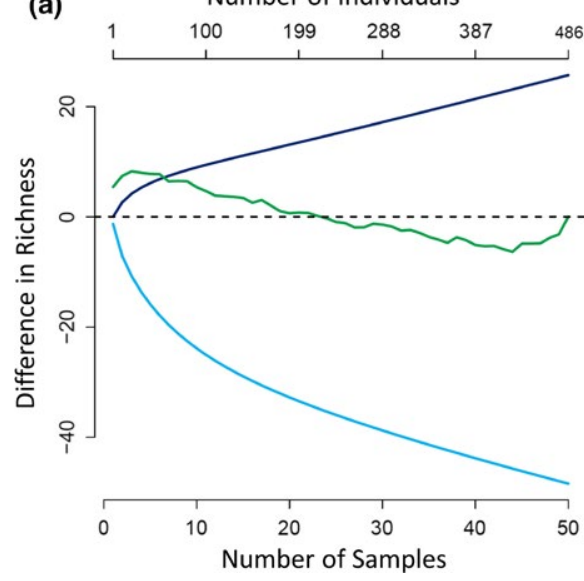

$\mathrm{N}$ effect $=$ Aggregation effect

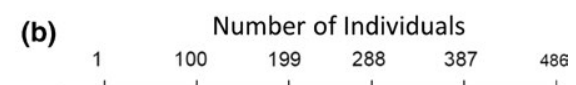

$\begin{array}{lllllll}\text { (b) } & 1 & 100 & 199 & 288 & 387 & 486\end{array}$

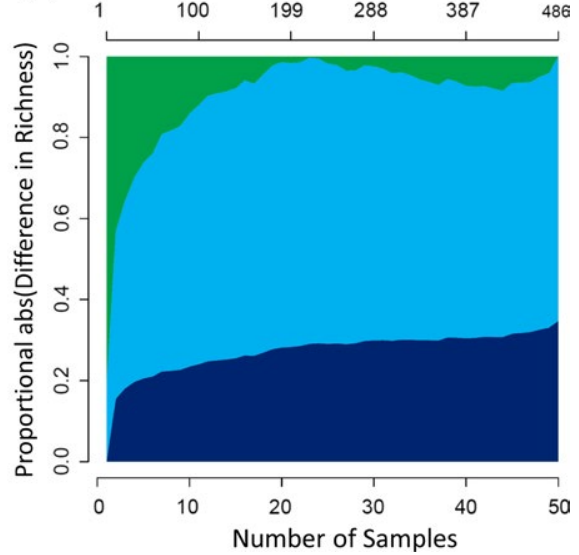

FIGURE 6 The effect of invasion on richness via individual effects on three components of community structure: SAD in dark blue, density in light blue, aggregation in green across scales. The raw differences (a) and proportional stacked absolute values (b). The $x$ axis represents sampling effort in both numbers of samples (i.e., plots) and individuals (see top axis). The rescaling between numbers of individuals and plots we carried out by defining the maximum number of individuals rarefied to (486 individuals) as equivalent to the maximum number of plots rarefied to (50 plots), other methods of rescaling are possible. In panel (a) the dashed black line indicates no change in richness

the interpretation of the link between change in condition and change in species richness.

The MoB framework that we have introduced here provides a comprehensive answer to this question by taking a spatially explicit approach and decomposing the effect of the condition (treatment) on richness into its individual components. The two-scale analysis provides a big-picture understanding of the differences and components of richness by only examining the single plot $(\alpha)$ and all plots combined $(\gamma)$ scales. The multiscale analysis expands the endeavor to cover a continuum of scales, and quantitatively decomposes change in richness into three components: change in the shape of the SAD, change in individual density, and change in spatial aggregation. As such, we cannot only quantify how richness changes at any scale of interest, but also identify how the change occurs and consequently push the ecological question to a more mechanistic 
level. For example, we can ask to what extent the effects on species richness are driven by numbers of individuals. Or instead, whether common and rare species, or their spatial distributions, are more strongly influenced by the treatments.

Here, we considered the scenario of comparing a discrete treatment effect on species richness, but clearly the MoB framework will need to be extended to other kinds of experimental and observational designs and questions (Supporting Information S6). The highest priority extension of the framework is to generalize it from a comparison of discrete treatment variables to continuous drivers such as temperature and productivity. Additionally, we recognize that abundance is difficult to collect for many organisms and that there is a need to understand if alternative measures of commonness (e.g., visual cover, biomass) can also be used to gain similar insights. Finally, we have only focused on taxonomic diversity here, whereas other types of biodiversity-most notably functional and phylogenetic diversity-are often of great interest, and comparisons such as those we have overviewed here would also be of great importance for these other biodiversity measures. Importantly, phylogenetic and functional diversity measures share many properties of taxonomic diversity that we have overviewed here (e.g., scale-dependence, nonlinear accumulations, rarefactions, etc.) (e.g., Chao, Chiu, \& Jost, 2014), and it would seem quite useful to extend our framework to these sorts of diversities. We look forward to working with the community to develop extensions of the MoB framework that are most needed for understanding scale dependence in diversity change and overcome the limitations of the framework as currently implemented (Supporting Information S6).

The MoB framework is a novel and robust approach that explicitly addresses the issue of scale-dependence in studies of diversity, and quantitatively disentangles diversity change into its three components. Our method demonstrates how spatially explicit community data and carefully framed comparisons can be combined to yield new insight into the underlying components of biodiversity. We hope the MoB framework will help ecologists move beyond single-scale analyses of simple and relatively uninformative metrics such as species richness alone. We view this as a critical step in reconciling much confusion and debate over the direction and magnitude of diversity responses to natural and anthropogenic drivers. Ultimately accurate predictions of biodiversity change will require knowledge of the relevant drivers and the spatial scales over which they are most relevant, which MoB (and its future extensions), helps to uncover.

\section{ACKNOWLEDGEMENTS}

This paper emerged from several workshops funded with the support (to JMC) from the German Centre for Integrative Biodiversity Research (iDiv) Halle-Jena-Leipzig funded by the German Research Foundation (FZT 118) and by the Alexander von Humboldt Foundation as part of the Alexander von Humboldt Professorship of TMK. DJM was also supported by College of Charleston startup funding. We further thank N. Sanders, J. Belmaker, D. Storch, N. Baker and the anonymous reviewers and associate editor for discussions and comments on our approach.

\section{AUTHORS' CONTRIBUTIONS}

D.J.M., X.X., J.M.C. and B.J.M. conceived the study and the overall approach, and all authors participated in multiple working group meetings to develop and refine the approach; J.M.C. and T.M.K. collected the data for the empirical example that led to Figures 3-6; D.J.M., X.X., F.M. and T.E. wrote the R package, N.J.G., J.M.C. and B.J.M. provided guidance on method development, D.J.M. carried out the analysis of the empirical example, and X.X. carried out the sensitivity analysis; D.J.M. and X.X. wrote the first draft of the manuscript, and all authors contributed substantially to revisions.

\section{DATA ACCESSIBILITY}

The code and data is archived with the $\mathrm{R}$ package on GitHub: https:// github.com/mobiodiv/mobr and the specific release of this code is also archived on Zenodo: https://zenodo.org/record/1434493\#. W6mUtmhKhnl (McGlinn et al., 2018).

\section{ORCID}

Daniel J. McGlinn (iD http://orcid.org/0000-0003-2359-3526

Felix May iD http://orcid.org/0000-0002-1106-8188

Thore Engel iD http://orcid.org/0000-0002-9245-4397

Shane A. Blowes iD http://orcid.org/0000-0001-6310-3670

Oliver Purschke iD http://orcid.org/0000-0003-0444-0882

\section{REFERENCES}

Arrhenius, O. (1921). Species and area. Journal of Ecology, 9, 95-99. https://doi.org/10.2307/2255763

Baddeley, A., Diggle, P. J., Hardegen, A., Lawrence, T., Milne, R. K., \& Nair, G. (2014). On tests of spatial pattern based on simulation envelopes. Ecological Monographs, 84, 477-489. https://doi. org/10.1890/13-2042.1

Blowes, S. A., Belmaker, J., \& Chase, J. M. (2017). Global reef fish richness gradients emerge from divergent and scale-dependent component changes. Proceedings of the Royal Society B, 284, 20170947. https:// doi.org/10.1098/rspb.2017.0947

Cayuela, L., Gotelli, N. J., \& Colwell, R. K. (2015). Ecological and biogeographic null hypotheses for comparing rarefaction curves. Ecological Monographs, 85, 437-455. https://doi.org/10.1890/14-1261.1

Chalcraft, D. R., Williams, J. W., Smith, M. D., \& Willig, M. R. (2004). Scale dependence in the species-richness-productivity relationship: The role of species turnover. Ecology, 85, 2701-2708. https://doi. org/10.1890/03-0561

Chao, A., Chiu, C. H., \& Jost, L. (2014). Unifying species diversity, phylogenetic diversity, functional diversity, and related similarity and differentiation measures through Hill numbers. Annual Review of Ecology, Evolution, and Systematics, 45, 297-324. https://doi.org/10.1146/ annurev-ecolsys-120213-091540

Chase, J. M., \& Knight, T. M. (2013). Scale-dependent effect sizes of ecological drivers on biodiversity: Why standardised sampling is not enough. Ecology Letters, 16, 17-26. https://doi.org/10.1111/ele.12112

Chase, J. M., \& Leibold, M. A. (2002). Spatial scale dictates the productivity-biodiversity relationship. Nature, 416, 427-430. https:// doi.org/10.1038/416427a 
Chase, J. M., McGill, B. J., McGlinn, D. J., May, F., Blowes, S. A., Xiao, X., ... Gotelli, N. J. (2018). Embracing scale-dependence to achieve a deeper understanding of biodiversity and its change across communities. Ecology Letters, 21, 1737-1751. https://doi.org/10.1111/ ele.13151

Chiarucci, A., Bacaro, G., Rocchini, D., Ricotta, C., Palmer, M. W., \& Scheiner, S. M. (2009). Spatially constrained rarefaction: Incorporating the autocorrelated structure of biological communities into sample-based rarefaction. Community Ecology, 10, 209-214. https://doi.org/10.1556/ComEc.10.2009.2.11

Collins, M. D., \& Simberloff, D. (2009). Rarefaction and nonrandom spatial dispersion patterns. Environmental and Ecological Statistics, 16, 89-103. https://doi.org/10.1007/s10651-007-0051-y

Fridley, J. D., Brown, R. L., \& Bruno, J. E. (2004). Null models of exotic invasion and scale-dependent patterns of native and exotic species richness. Ecology, 85, 3215-3222. https://doi.org/10.1890/03-0676

Gotelli, N. J., \& Colwell, R. K. (2001). Quantifying biodiversity Procedures and pitfalls in the measurement and comparison of species richness. Ecology Letters, 4, 379-391. https://doi. org/10.1046/j.1461-0248.2001.00230.x

Harte, J. (2011). Maximum entropy and ecology: A theory of abundance, distribution, and energetics. New York: Oxford University Press Inc. https://doi.org/10.1093/acprof:oso/9780199593415.001.0001

Harte, J., Zillio, T., Conlisk, E., \& Smith, A. B. (2008). Maximum entropy and the state-variable approach to macroecology. Ecology, 89, 27002711. https://doi.org/doi:10.1890/07-1369.1

He, F. L., \& Legendre, P. (2002). Species diversity patterns derived from species-area models. Ecology, 83, 1185-1198.

Hubbell, S. P. (2001). The unified neutral theory of biodiversity and biogeography. Princeton, NJ: Princeton University Press.

Hurlbert, S. H. (1971). The nonconcept of species diversity: A critique and alternative parameters. Ecology, 52, 577-586. https://doi. org/10.2307/1934145

Karlson, R. H., Cornell, H. V., \& Hughes, T. P. (2007). Aggregation influences coral species richness at multiple spatial scales. Ecology, 88, 170-177. https://doi.org/10.1890/0012-9658(2007)88[170:AICSRA]2.0.CO;2

Knight,K.S., \& Reich,P.B.(2005).Oppositerelationshipsbetween invasibility and native species richness at patch versus landscape scales. Oikos, 109, 81-88. https://doi.org/10.1111/j.0030-1299.2005.13639.x

Legendre, P., \& Legendre, L. (2012). Numerical ecology (3rd English edn). Boston, MA: Elsevier.

Leibold, M. A., \& Chase, J. M. (2017). Metacommunity ecology. Princeton, NJ: Princeton University Press. https://doi.org/10.2307/j.ctt1wf4d24

Loosmore, N. B., \& Ford, E. D. (2006). Statistical inference using the G or K point pattern spatial statistics. Ecology, 87, 1925-1931. https://doi. org/10.1890/0012-9658(2006) 87[1925:SIUTGO]2.0.CO;2

May, F., Gerstner, K., McGlinn, D. J., Xiao, X., \& Chase, J. M. (2018). mobsim: An R package for the simulation and measurement of biodiversity across spatial scales. Methods in Ecology and Evolution, 9, 14011408. https://doi.org/10.1111/2041-210X.12986

McGill, B. J. (2010). Towards a unification of unified theories of biodiversity. Ecology Letters, 13, 627-642. https://doi. org/10.1111/j.1461-0248.2010.01449.x

McGill, B. J. (2011a). Linking biodiversity patterns by autocorrelated random sampling. American Journal of Botany, 98, 481-502. https://doi. org/10.3732/ajb.1000509

McGill, B. J. (2011b). Species abundance distributions. In A. E. Magurran \& B. J. McGill (Eds.), In Biological diversity: Frontiers in measurement and assessment (pp. 105-122). Oxford, UK: Oxford University Press.

McGlinn, D. J., \& Palmer, M. W. (2009). Modeling the sampling effect in the species-time-area relationship. Ecology, 90, 836-846. https://doi. org/10.1890/08-0377.1

McGlinn, D. J., Xiao, X., Kitzes, J., \& White, E. P. (2015). Exploring the spatially explicit predictions of the Maximum Entropy Theory of
Ecology. Global Ecology and Biogeography, 24, 675-684. https://doi. org/10.1111/geb.12295

McGlinn, D.J.,Xiao,X., May, F.,Engel,T.,\&Oliver,C.(2018).mobr:Measurement of Biodiversity in R. https://doi.org/10.5281/zenodo.1434493

Olszewski, T. D. (2004). A unified mathematical framework for the measurement of richness and evenness within and among multiple communities. Oikos, 104, 377-387. https://doi. org/10.1111/j.0030-1299.2004.12519.x

Palmer, M. W., McGlinn, D. J., \& Fridley, J. F. (2008). Artifacts and artifictions in biodiversity research. Folia Geobotanica, 43, 245-257. https://doi.org/10.1007/s12224-008-9012-y

Palmer, M. W., \& van der Maarel, E. (1995). Variance in species richness, species association, and niche limitation. Oikos, 73, 203-213. https:// doi.org/10.2307/3545909

Powell, K. I., Chase, J. M., \& Knight, T. M. (2013). Invasive plants have scale-dependent effects on diversity by altering species-area relationships. Science, 339, 316-318. https://doi.org/10.1126/ science.1226817

Rosenzweig, M. L. (1995). Species diversity in space and time. Cambridge, UK: Cambridge University Press. https://doi.org/10.1017/ CBO9780511623387

Scheiner, S. M., Cox, S. B., Willig, M., Mittelbach, G. G., Osenberg, C., \& Kaspari, M. (2000). Species richness, species-area curves and Simpson's paradox. Evolutionary Ecology Research, 2, 791-802.

Simberloff, D. (1972). Properties of the rarefaction diversity measurement. The American Naturalist, 106, 414-418. https://doi. org/10.1086/282781

Srivastava, D. S., \& Lawton, J. H. (1998). Why more productive sites have more species: An experimental test of theory using tree-hole communities. The American Naturalist, 152, 510-529. https://doi. org/10.1086/286187

Supp, S. R., Xiao, X., Ernest, S. K. M., \& White, E. P. (2012). An experimental test of the response of macroecological patterns to altered species interactions. Ecology, 93, 2505-2511. https://doi. org/10.1890/12-0370.1

Tilman, D. (1994). Competition and biodiversity in spatially structured habitats. Ecology, 75, 2-16. https://doi.org/10.2307/1939377

Tjørve, E., Kunin, W. E., Polce, C., \& Tjørve, K. M. C. (2008). Speciesarea relationship: Separating the effects of species abundance and spatial distribution. Journal of Ecology, 96, 1141-1151. https://doi. org/10.1111/j.1365-2745.2008.01433.x

Ulrich, W., \& Gotelli, N. J. (2010). Null model analysis of species associations using abundance data. Ecology, 91, 3384-3397. https://doi.org/ doi:10.1890/09-2157.1

Vellend, M. (2016). The theory of ecological communities. Princeton, NJ: PrincetonUniversity Press.https://doi.org/10.1515/9781400883790

Williams, C. B. (1943). Area and number of species. Nature, 152, 264-267. https://doi.org/10.1038/152264a0

\section{SUPPORTING INFORMATION}

Additional supporting information may be found online in the Supporting Information section at the end of the article.

How to cite this article: McGlinn DJ, Xiao X, May F, et al. Measurement of Biodiversity (MoB): A method to separate the scale-dependent effects of species abundance distribution, density, and aggregation on diversity change. Methods Ecol Evol. 2019;10:258-269. https://doi.org/10.1111/2041-210X.13102 